\title{
Jordan $\tau$-derivations of Prime GPI-rings
}

\author{
Jheng-Huei Lin
}

\begin{abstract}
Let $R$ be a noncommutative prime ring, with maximal symmetric ring of quotients $Q_{m s}(R)$ and extended centriod $C$, and let $\tau$ be an anti-automorphism of $R$. An additive map $\delta: R \rightarrow Q_{m s}(R)$ is called a Jordan $\tau$-derivation if $\delta\left(x^{2}\right)=$ $\delta(x) x^{\tau}+x \delta(x)$ for all $x \in R$. In 2015 Lee and the author proved that any Jordan $\tau$-derivation of $R$ is X-inner if either $R$ is not a GPI-ring or $R$ is a PI-ring except when $\operatorname{char} R=2$ and $\operatorname{dim}_{C} R C=4$. In the paper we prove that, when $R$ is a prime GPI-ring but is not a PI-ring, any Jordan $\tau$-derivation is X-inner if either $\tau$ is of the second kind or both char $R \neq 2$ and $\tau$ is of the first kind with $\operatorname{deg} \tau^{2} \neq 2$.
\end{abstract}

\section{Introduction}

Throughout the paper, $R$ is a prime ring with center $Z(R)$. Let $Q_{m l}(R)\left(\operatorname{resp} . Q_{m s}(R)\right)$ be the maximal left (resp. symmetric) ring of quotients of $R$, and let $Q_{s}(R)$ be the Martindale symmetric ring of quotients of $R$. The center $C$ of $Q_{m l}(R)$ is called the extended centroid of $R$. Note that $Q_{s}(R) \subseteq Q_{m s}(R) \subseteq Q_{m l}(R)$ and $Z\left(Q_{m s}(R)\right)=Z\left(Q_{s}(R)\right)=C$. We refer the readers to see [2] for more details.

By a derivation (resp. Jordan derivation) of $R$ we mean an additive map $\delta: R \rightarrow$ $Q_{m l}(R)$ satisfying $\delta(x y)=\delta(x) y+x \delta(y)$ for all $x, y \in R\left(\operatorname{resp} . \delta\left(x^{2}\right)=\delta(x) x+x \delta(x)\right.$ for all $x \in R$ ). Obviously, a derivation is a Jordan derivation, but, in general, the converse is not true. Herstein [9], Lee, and the author 15] characterized Jordan derivations of prime rings. They showed that an additive map $\delta: R \rightarrow Q_{m l}(R)$ is a Jordan derivation if and only if it is of the form $d+\mu$, where $d: R \rightarrow Q_{m l}(R)$ is a derivation and $\mu: R \rightarrow C$ is an additive map satisfying $2 \mu=0$ and $\mu\left(x^{2}\right)=0$ for all $x \in R$. Moreover, Cusack [6] and Brešar [3] independently generalized Herstein's result, i.e., they proved that every Jordan derivation of a 2 -torsion free semi-prime ring is a derivation.

Let $\tau: R \rightarrow R$ be an anti-automorphism of $R$. An additive map $\delta: R \rightarrow Q_{m s}(R)$ is called a Jordan $\tau$-derivation of $R$ if $\delta\left(x^{2}\right)=x \delta(x)+\delta(x) x^{\tau}$ for all $x \in R$. A Jordan $\tau$ derivation $\delta$ of $R$ is said to be inner (resp. X-inner) if there exists $a \in R$ (resp. $a \in Q_{m s}(R)$ ) such that $\delta(x)=a x^{\tau}-x a$ for $x \in R$.

Received October 14, 2019; Accepted November 14, 2019.

Communicated by Ching Hung Lam.

2010 Mathematics Subject Classification. 16R60, 16N60, 16W10, 16W25.

Key words and phrases. prime GPI-ring, PI-ring, (X-inner) Jordan $\tau$-derivation, anti-automorphism, functional identity, maximal symmetric ring of quotients. 
Let $A$ be a unital ring. Suppose that $*: A \rightarrow A$ is an involution of $A$, i.e., $*$ is an antiautomorphism of $A$ such that $\left(x^{*}\right)^{*}=x$ for all $x \in A$. The problem of the representability of quadratic forms by bilinear forms is connected with the structure of Jordan $*$-derivations (see 18,19]). Brešar and Vukman showed that if a unital *-ring $A$ contains $1 / 2$ and a central invertible skew-hermitian element $\mu$ (i.e., $\mu^{*}=-\mu$ ), then every Jordan $*$-derivation $\delta: A \rightarrow A$ is inner (see [4, Theorem 1]). In particular, every Jordan $*$-derivation $\delta: A \rightarrow A$ of a unital complex *-algebra $A$ is inner because any complex $*$-algebra $A$ always assumes $(\beta x)^{*}=\bar{\beta} x^{*}$ for all $x \in A$ and $\beta \in \mathbb{C}$.

Let $H$ be a real Hilbert space with $\operatorname{dim}_{\mathbb{R}} H>1$. Let $\mathcal{B}(H)$ stand for the algebra of all bounded linear operators on the Hilbert space $H$ and let $\mathcal{A}$ be a standard operator algebra on $H$. Then $\mathcal{B}(H)$ can be endowed with a canonical involution, say $*$. It is known that $\mathcal{A}$ is a prime algebra over $\mathbb{R}$ with nonzero socle. Moreover, $Q_{m s}(\mathcal{A})=Q_{m s}(\mathcal{B}(H))=\mathcal{B}(H)$ (see 5. Theorem 1.3]). Semrl proved that every Jordan $*$-derivation $\delta: \mathcal{B}(H) \rightarrow \mathcal{B}(H)$ is inner (see [17]), and that all Jordan $*$-derivations $\delta: \mathcal{A} \rightarrow \mathcal{B}(H)$ are X-inner (see [20]). Chuang et al. extended Šemrl's theorems above by proving the theorem: Let $R$ be a prime ring, which is not a division ring. Let $\tau$ be an anti-automorphism of $R$ and let $\delta: R \rightarrow Q_{s}(R)$ be a Jordan $\tau$-derivation. If char $R \neq 2$ and the socle of $R$ is nonzero, then $\delta$ is X-inner (see 5, Theorem 1.2]). Moreover, the structure of Jordan $*$-derivations of prime rings is completely determined. To be precise, it was proved that every Jordan *-derivation of $R$ is $\mathrm{X}$-inner except when char $R=2$ and $\operatorname{dim}_{C} R C=4$ (see [7, 13, 14]). Note that there exist non X-inner Jordan $*$-derivations when $\operatorname{char} R=2$ and $\operatorname{dim}_{C} R C=4$ (see [13, Theorem 3.1]).

The reader is referred to [2] for the definitions of PI-rings and GPI-rings. In [16], Lee and the author showed that any Jordan $\tau$-derivation of $R$ is $\mathrm{X}$-inner if either $R$ is not a GPI-ring or $R$ is a PI-ring except when char $R=2$ and $\operatorname{dim}_{C} R C=4$. In order to completely characterize Jordan $\tau$-derivations of $R$, they raised the following question.

Question 1.1. Let $R$ be a prime GPI-ring, which is not commutative, with an antiautomorphism $\tau$. Suppose that neither $R$ is a PI-ring nor $R$ is a division ring. Is any Jordan $\tau$-derivation of $R$ X-inner?

We remark that, by Martindale's theorem [10, Theorem 3], if $R$ is both a prime GPIring and a division ring, then it is a PI-ring and Question 1.1 is solved by [16, Theorem 2.9] in this case. Hence Question 1.1 is reduced to the case that $R$ is a prime GPI-ring but is not a PI-ring. Given an automorphism (resp. anti-automorphism) $g$ of $R, g$ can be uniquely extended to an automorphism (resp. anti-automorphism) of $Q_{s}(R)$ (see [2, Proposition 2.5.3] for the automorphism case and [2, Proposition 2.5.4] for the anti-automorphism case). An automorphism (resp. anti-automorphism) $g$ is said to be of the first kind if $\beta^{g}=\beta$ for all $\beta \in C$. Otherwise, $g$ is said to be of the second kind. We first give an affirmative answer 
to Question 1.1 when $\tau$ is of the second kind.

Theorem 1.2. Let $R$ be a noncommutative prime ring with an anti-automorphism $\tau$. If $\tau$ is of the second kind, then any Jordan $\tau$-derivation of $R$ is $X$-inner except when char $R=2$ and $\operatorname{dim}_{C} R C=4$.

We next consider the case that $\tau$ is of the first kind. By an X-inner automorphism we mean an automorphism of the form $x \mapsto u x u^{-1}$ for all $x \in R$, where $u \in Q_{s}(R)$. Kharchenko proved that, given an automorphism $\sigma$ of a prime GPI-ring, if $\sigma$ is of the first kind, then it is X-inner (see [8, Proof of Proposition 2]). By Kharchenko's theorem, $\tau^{2}$ is X-inner when $R$ is a prime GPI-ring and $\tau$ is of the first kind. The complexity of the question depends on that of $\tau^{2}$.

Definition 1.3. Let $R$ be a prime GPI-ring with an automorphism $\sigma$ of the first kind. Then there exists $u \in Q_{s}(R)$ such that $x^{\sigma}=u x u^{-1}$ for all $x \in R$. We say that $\operatorname{deg} \sigma=m$ if $u$ is algebraic of minimal degree $m$ over $C$. Moreover, $\operatorname{deg} \sigma=\infty$ if $u$ is not algebraic over $C$.

Clearly, $\operatorname{deg} \sigma$ is independent of the element $u$ we choose. Also, if $\operatorname{deg} \tau^{2}=1$, then $\tau$ is an involution and Question 1.1 has been solved in [13, Theorem 1.2]. The following is the second main theorem of the paper.

Theorem 1.4. Let $R$ be a prime GPI-ring with char $R \neq 2$ and an anti-automorphism $\tau$ of the first kind. If $\operatorname{deg} \tau^{2} \neq 2$, then any Jordan $\tau$-derivation of $R$ is $X$-inner.

We remark that the case of $\operatorname{deg} \tau^{2}=2$ keeps unknown.

\section{Proof of Theorem 1.2}

Throughout the section, $R$ is a prime ring with an anti-automorphism $\tau$ of the second kind. To characterize Jordan $\tau$-derivations of $R$, we need some results concerning functional identities. In [12], Lee dealt with functional identities on prime rings with an automorphism. For our purpose, we will follow his viewpoint to get useful results about functional identities.

We first introduce some notations. For maps $f: R^{r-1} \rightarrow Q_{m l}(R)$ and $g: R^{r-2} \rightarrow$ $Q_{m l}(R)$, we write

$$
f^{i}\left(\bar{x}_{r}\right)=f\left(x_{1}, \ldots, x_{i-1}, x_{i+1}, \ldots, x_{r}\right)
$$

and

$$
g^{i j}\left(\bar{x}_{r}\right)=g^{j i}\left(\bar{x}_{r}\right)=g\left(x_{1}, \ldots, x_{i-1}, x_{i+1}, \ldots, x_{j-1}, x_{j+1}, \ldots, x_{r}\right)
$$

where $\bar{x}_{r}=\left(x_{1}, \ldots, x_{r}\right) \in R^{r}$ and $1 \leq i<j \leq r$. Our purpose is to prove the following theorem. 
Theorem 2.1. Let $R$ be a prime ring with an anti-automorphism $\tau$ of the second kind. Suppose that $E_{i t}, F_{\ell 1}: R^{r-1} \rightarrow Q_{m l}(R)$ are $(r-1)$-additive maps such that

$$
\sum_{i=1}^{r} E_{i 1}^{i}\left(\bar{x}_{r}\right) x_{i}+\sum_{i=1}^{r} E_{i 2}^{i}\left(\bar{x}_{r}\right) x_{i}^{\tau}+\sum_{\ell=1}^{r} x_{\ell} F_{\ell 1}^{\ell}\left(\bar{x}_{r}\right) \in C
$$

for $\bar{x}_{r} \in R^{r}$, where $1 \leq i, \ell \leq r$ and $t=1,2$. If $R$ is not a PI-ring, then there exist a nonzero ideal $I$ of $R,(r-2)$-additive maps $p_{i t \ell 1}: I^{r-2} \rightarrow Q_{m l}(R)$, and $(r-1)$-additive maps $\lambda_{i 1}: I^{r-1} \rightarrow C$ such that

$$
E_{i 1}^{i}\left(\bar{x}_{r}\right)=\sum_{\substack{1 \leq \ell \leq r \\ \ell \neq i}} x_{\ell} p_{i 1 \ell 1}^{i \ell}\left(\bar{x}_{r}\right)+\lambda_{i 1}^{i}\left(\bar{x}_{r}\right), \quad E_{i 2}^{i}\left(\bar{x}_{r}\right)=\sum_{\substack{1 \leq \ell \leq r \\ \ell \neq i}} x_{\ell} p_{i 2 \ell 1}^{i \ell}\left(\bar{x}_{r}\right)
$$

and

$$
F_{\ell 1}^{\ell}\left(\bar{x}_{r}\right)=-\sum_{\substack{1 \leq i \leq r \\ i \neq \ell}} p_{i 1 \ell 1}^{i \ell}\left(\bar{x}_{r}\right) x_{i}-\sum_{\substack{1 \leq i \leq r \\ i \neq \ell}} p_{i 2 \ell 1}^{i \ell}\left(\bar{x}_{r}\right) x_{i}^{\tau}-\lambda_{\ell 1}^{\ell}\left(\bar{x}_{r}\right)
$$

for all $\bar{x}_{r} \in I^{r}$, where $1 \leq i, \ell \leq r$ and $t=1,2$.

Corollary 2.2. Let $R$ be a prime ring with an anti-automorphism $\tau$ of the second kind. Suppose that $E_{i}, F_{\ell}: R^{r-1} \rightarrow Q_{m l}(R)$ are $(r-1)$-additive maps such that

$$
\sum_{i=1}^{r} E_{i}^{i}\left(\bar{x}_{r}\right) x_{i}^{\tau}+\sum_{\ell=1}^{r} x_{\ell} F_{\ell}^{\ell}\left(\bar{x}_{r}\right) \in C
$$

for $\bar{x}_{r} \in R^{r}$, where $1 \leq i, \ell \leq r$. If $R$ is not a PI-ring, then there exist a nonzero ideal $I$ of $R$ and $(r-2)$-additive maps $p_{i \ell}: I^{r-2} \rightarrow Q_{m l}(R)$ such that

$$
E_{i}^{i}\left(\bar{x}_{r}\right)=\sum_{\substack{1 \leq \ell \leq r \\ \ell \neq i}} x_{\ell} p_{i \ell}^{i \ell}\left(\bar{x}_{r}\right) \quad \text { and } \quad F_{\ell}^{\ell}\left(\bar{x}_{r}\right)=-\sum_{\substack{1 \leq i \leq r \\ i \neq \ell}} p_{i \ell}^{i \ell}\left(\bar{x}_{r}\right) x_{i}^{\tau}
$$

for all $\bar{x}_{r} \in I^{r}$, where $1 \leq i, \ell \leq r$.

Proof. By Theorem 2.1, there exist a nonzero ideal $I$ of $R,(r-2)$-additive maps $p_{i t \ell 1}: I^{r-2}$ $\rightarrow Q_{m l}(R)$, and $(r-1)$-additive maps $\lambda_{i 1}: I^{r-1} \rightarrow C$ such that

$$
0=\sum_{\substack{1 \leq \ell \leq r \\ \ell \neq i}} x_{\ell} p_{i 1 \ell 1}^{i \ell}\left(\bar{x}_{r}\right)+\lambda_{i 1}^{i}\left(\bar{x}_{r}\right), \quad E_{i}^{i}\left(\bar{x}_{r}\right)=\sum_{\substack{1 \leq \ell \leq r \\ \ell \neq i}} x_{\ell} p_{i 2 \ell 1}^{i \ell}\left(\bar{x}_{r}\right),
$$

and

$$
F_{\ell}^{\ell}\left(\bar{x}_{r}\right)=-\sum_{\substack{1 \leq i \leq r \\ i \neq \ell}} p_{i 1 \ell 1}^{i \ell}\left(\bar{x}_{r}\right) x_{i}-\sum_{\substack{1 \leq i \leq r \\ i \neq \ell}} p_{i 2 \ell 1}^{i \ell}\left(\bar{x}_{r}\right) x_{i}^{\tau}-\lambda_{\ell 1}^{\ell}\left(\bar{x}_{r}\right)
$$

for all $\bar{x}_{r} \in I^{r}$, where $1 \leq i, \ell \leq r$ and $t=1,2$. In view of [1, Theorem 2.4], $p_{i 1 \ell 1}=0$ and $\lambda_{i 1}=0$ for $1 \leq i, \ell \leq r$. The proof is complete by putting $p_{i \ell}=p_{i 2 \ell 1}$. 
To begin the proof of Theorem 2.1, we first give the following lemma.

Lemma 2.3. Suppose that $E_{i}, F_{\ell}: R^{r-1} \rightarrow Q_{m l}(R)$ are $(r-1)$-additive maps such that

$$
\sum_{i=1}^{r} E_{i}^{i}\left(\bar{x}_{r}\right) x_{i}+\sum_{\ell=1}^{r} F_{\ell}^{\ell}\left(\bar{x}_{r}\right) x_{\ell}^{\tau} \in C
$$

for $\bar{x}_{r} \in R^{r}$, where $1 \leq i, \ell \leq r$. If $R$ is not a PI-ring, then there exists a nonzero ideal $I$ of $R$ such that $E_{i}^{i}=0=F_{\ell}^{\ell}$ on $I^{r}$ for $1 \leq i, \ell \leq r$.

Before proving it, we define the following notation (see $\left[12\right.$ ). For a map $f: R^{r-1} \rightarrow$ $Q_{m l}(R)$ and $t \neq i$, we write

$$
f^{i}\left(\bar{x}_{r} ;\{y\}_{t}\right)=f\left(z_{1}, \ldots, z_{i-1}, z_{i+1}, \ldots, z_{r}\right)
$$

where $z_{t}=y$ and $z_{j}=x_{j}$ for $j \neq t$, i.e., we replace $x_{t}$ by $y$ in $f^{i}\left(\bar{x}_{r}\right)$.

Proof of Lemma 2.3. Let $A:=\{1,2, \ldots, r\}$ and

$$
L:=\left\{\ell \in A \mid \text { there exists a nonzero ideal } J \text { of } R \text { such that } F_{\ell}^{\ell}=0 \text { on } J^{r}\right\} .
$$

We proceed the proof by induction on $r-|L|$.

Suppose first that $r-|L|=0$, i.e., $L=A$. Then there exists a nonzero ideal $J$ such that $F_{\ell}^{\ell}=0$ on $J^{r}$ for all $\ell=1, \ldots, r$. Thus $\sum_{i=1}^{r} E_{i}^{i}\left(\bar{x}_{r}\right) x_{i} \in C$ for all $\bar{x}_{r} \in J^{r}$. By [1, Theorem 2.4], $E_{i}^{i}=0$ on $J^{r}$ for all $i=1, \ldots, r$, as asserted.

Suppose next that $r-|L| \geq 1$. Without loss of generality, we may assume that $r \notin L$. Then, for any nonzero ideal $U$ of $R, F_{r}^{r} \neq 0$ on $U^{r}$. Fix $\beta \in C$ with $\beta^{\tau} \neq \beta$ and choose a nonzero ideal $K$ of $R$ such that $\beta K \subseteq R$. Then, by (2.2), we have

$$
\begin{aligned}
& \sum_{i=1}^{r-1}\left(E_{i}^{i}\left(\bar{x}_{r} ;\left\{\beta x_{r}\right\}_{r}\right)-\beta E_{i}^{i}\left(\bar{x}_{r}\right)\right) x_{i} \\
+ & \sum_{\ell=1}^{r-1}\left(F_{\ell}^{\ell}\left(\bar{x}_{r} ;\left\{\beta x_{r}\right\}_{r}\right)-\beta F_{\ell}^{\ell}\left(\bar{x}_{r}\right)\right) x_{\ell}^{\tau}+\left(\beta^{\tau}-\beta\right) F_{r}^{r}\left(\bar{x}_{r}\right) x_{r}^{\tau} \in C
\end{aligned}
$$

for all $\bar{x}_{r} \in K^{r}$. Let $K_{1}=K \cap K^{\tau}$. Then $K_{1}$ is an ideal of $R$ such that $K_{1}^{\tau^{-1}} \subseteq K$ and, by $(2.3)$, we have

$$
\sum_{i=1}^{r-1} \widetilde{E}_{i}^{i}\left(\bar{x}_{r}\right) x_{i}+F_{r}^{r}\left(\bar{x}_{r}\right) x_{r}+\sum_{\ell=1}^{r-1} \widetilde{F}_{\ell}^{\ell}\left(\bar{x}_{r}\right) x_{\ell}^{\tau} \in C
$$

for all $\bar{x}_{r} \in K_{1}^{r}$, where

$$
\widetilde{E}_{i}^{i}\left(\bar{x}_{r}\right)=\left(\beta^{\tau}-\beta\right)^{-1}\left(E_{i}^{i}\left(\bar{x}_{r} ;\left\{\beta x_{r}^{\tau^{-1}}\right\}_{r}\right)-\beta E_{i}^{i}\left(\bar{x}_{r} ;\left\{x_{r}^{\tau^{-1}}\right\}_{r}\right)\right)
$$


and

$$
\widetilde{F}_{\ell}^{\ell}\left(\bar{x}_{r}\right)=\left(\beta^{\tau}-\beta\right)^{-1}\left(F_{\ell}^{\ell}\left(\bar{x}_{r} ;\left\{\beta x_{r}^{\tau^{-1}}\right\}_{r}\right)-\beta F_{\ell}^{\ell}\left(\bar{x}_{r} ;\left\{x_{r}^{\tau^{-1}}\right\}_{r}\right)\right)
$$

Set

$$
L_{1}:=\left\{\ell \mid 1 \leq \ell \leq r-1 \text {, there exists a nonzero ideal } J \text { of } R \text { such that } \widetilde{F}_{\ell}^{\ell}=0 \text { on } J^{r}\right\} .
$$

Let $\ell \in L$. Then $1 \leq \ell \leq r-1$ and there is a nonzero ideal $N$ of $R$ such that $F_{\ell}^{\ell}=0$ on $N^{r}$. By the definition of $\widetilde{F}_{\ell}^{\ell}$, there exists a nonzero ideal $M$ of $R$ contained in $N$ such that $\widetilde{F}_{\ell}^{\ell}=0$ on $M^{r}$ and so $\ell \in L_{1}$. Thus $|L| \leq\left|L_{1}\right|$ and $r-|L| \geq r-\left|L_{1}\right|>(r-1)-\left|L_{1}\right|$. By applying the induction hypothesis on (2.4), we have $F_{\ell}^{\ell}=0$ on $W^{r}$ for some nonzero ideal $W$ of $R$, a contradiction.

Proof of Theorem 2.1. Let $A:=\{1,2, \ldots, r\}$ and

$$
L:=\left\{i \in A \mid \text { there exists a nonzero ideal } J \text { of } R \text { such that } E_{i 2}^{i}=0 \text { on } J^{r}\right\} .
$$

We proceed the proof by induction on $r-|L|$.

Assume first that $r-|L|=0$, i.e., $L=A$. Then $E_{i 2}^{i}=0$ on $U^{r}$ for some nonzero ideal $U$ of $R$ and so (2.1) becomes

$$
\sum_{i=1}^{r} E_{i 1}^{i}\left(\bar{x}_{r}\right) x_{i}+\sum_{\ell=1}^{r} x_{\ell} F_{\ell 1}^{\ell}\left(\bar{x}_{r}\right) \in C
$$

for $\bar{x}_{r} \in U^{r}$. Hence the result follows from [1, Corollary 2.11].

Assume next that $r-|L| \geq 1$. Without loss of generality, assume that $r \notin L$. Then $E_{r 2}^{r} \neq 0$ on any nonzero ideal of $R$. Let $\beta \in C$ with $\beta^{\tau} \neq \beta$ and choose a nonzero ideal $J$ of $R$ such that $\beta J \subseteq R$. Then, by (2.1), we have

$$
\begin{aligned}
& \sum_{i=1}^{r-1}\left(E_{i 1}^{i}\left(\bar{x}_{r} ;\left\{\beta x_{r}\right\}_{r}\right)-\beta E_{i 1}^{i}\left(\bar{x}_{r}\right)\right) x_{i} \\
+ & \sum_{i=1}^{r-1}\left(E_{i 2}^{i}\left(\bar{x}_{r} ;\left\{\beta x_{r}\right\}_{r}\right)-\beta E_{i 2}^{i}\left(\bar{x}_{r}\right)\right) x_{i}^{\tau}+E_{r 2}^{r}\left(\bar{x}_{r}\right)\left(\beta^{\tau}-\beta\right) x_{r}^{\tau} \\
+ & \sum_{\ell=1}^{r-1} x_{\ell}\left(F_{\ell 1}^{\ell}\left(\bar{x}_{r} ;\left\{\beta x_{r}\right\}_{r}\right)-\beta F_{\ell 1}^{\ell}\left(\bar{x}_{r}\right)\right) \in C
\end{aligned}
$$

for all $\bar{x}_{r} \in J^{r}$. Let

$$
\begin{aligned}
& \widetilde{E}_{i 1}^{i}\left(\bar{x}_{r}\right)=\left(\beta^{\tau}-\beta\right)^{-1}\left(E_{i 1}^{i}\left(\bar{x}_{r} ;\left\{\beta x_{r}\right\}_{r}\right)-\beta E_{i 1}^{i}\left(\bar{x}_{r}\right)\right), \\
& \widetilde{E}_{i 2}^{i}\left(\bar{x}_{r}\right)=\left(\beta^{\tau}-\beta\right)^{-1}\left(E_{i 2}^{i}\left(\bar{x}_{r} ;\left\{\beta x_{r}\right\}_{r}\right)-\beta E_{i 2}^{i}\left(\bar{x}_{r}\right)\right),
\end{aligned}
$$

and

$$
\widetilde{F}_{\ell 1}^{\ell}\left(\bar{x}_{r}\right)=\left(\beta^{\tau}-\beta\right)^{-1}\left(F_{\ell 1}^{\ell}\left(\bar{x}_{r} ;\left\{\beta x_{r}\right\}_{r}\right)-\beta F_{\ell 1}^{\ell}\left(\bar{x}_{r}\right)\right) .
$$


Then

$$
\sum_{i=1}^{r-1} \widetilde{E}_{i 1}^{i}\left(\bar{x}_{r}\right) x_{i}+\sum_{i=1}^{r-1} \widetilde{E}_{i 2}^{i}\left(\bar{x}_{r}\right) x_{i}^{\tau}+E_{r 2}^{r}\left(\bar{x}_{r}\right) x_{r}^{\tau}+\sum_{\ell=1}^{r-1} x_{\ell} \widetilde{F}_{\ell 1}^{\ell}\left(\bar{x}_{r}\right) \in C
$$

for all $\bar{x}_{r} \in J^{r}$. Choose a nonzero ideal $J_{1}$ of $R$ contained in $J$ so that $J_{1}^{\tau^{-1}} \subseteq J$. By (2.5), we have

$$
\begin{aligned}
& \sum_{i=1}^{r-1} \widetilde{E}_{i 1}^{i}\left(\bar{x}_{r} ;\left\{x_{r}^{\tau^{-1}}\right\}_{r}\right) x_{i}+\sum_{i=1}^{r-1} \widetilde{E}_{i 2}^{i}\left(\bar{x}_{r} ;\left\{x_{r}^{\tau^{-1}}\right\}_{r}\right) x_{i}^{\tau}+E_{r 2}^{r}\left(\bar{x}_{r}\right) x_{r} \\
+ & \sum_{\ell=1}^{r-1} x_{\ell} \widetilde{F}_{\ell 1}^{\ell}\left(\bar{x}_{r} ;\left\{x_{r}^{\tau^{-1}}\right\}_{r}\right) \in C
\end{aligned}
$$

for all $\bar{x}_{r} \in J_{1}^{r}$. Set $G_{i 2}^{i}\left(\bar{x}_{r}\right):=\widetilde{E}_{i 2}^{i}\left(\bar{x}_{r} ;\left\{x_{r}^{\tau^{-1}}\right\}_{r}\right)$ and

$$
L_{1}:=\left\{i \mid 1 \leq i \leq r-1 \text {, there exists a nonzero ideal } J \text { of } R \text { such that } G_{i 2}^{i}=0 \text { on } J^{r}\right\} .
$$

Let $i \in L$ and $i \neq r$. Then there exists a nonzero ideal $N$ of $R$ such that $E_{i 2}^{i}=0$ on $N^{r}$. From the definition of $G_{i 2}^{i}$, there is a nonzero ideal $M$ of $R$ contained in $N$ such that $G_{i 2}^{i}=0$ on $M^{r}$, and so $i \in L_{1}$. Thus

$$
r-|L| \geq r-\left|L_{1}\right|>(r-1)-\left|L_{1}\right|
$$

By the induction hypothesis, there exist a nonzero ideal $J_{2}$ of $R$ contained in $J_{1}$ and $(r-2)$-additive maps $p_{r 2 \ell 1}: J_{2}^{r-2} \rightarrow Q_{m l}(R)$ such that

$$
E_{r 2}^{r}\left(\bar{x}_{r}\right)=\sum_{\ell=1}^{r-1} x_{\ell} p_{r 2 \ell 1}^{r \ell}\left(\bar{x}_{r}\right)
$$

for all $\bar{x}_{r} \in J_{2}^{r}$. Substituting it into (2.1), we have

$$
\sum_{i=1}^{r} E_{i 1}^{i}\left(\bar{x}_{r}\right) x_{i}+\sum_{i=1}^{r-1} E_{i 2}^{i}\left(\bar{x}_{r}\right) x_{i}^{\tau}+\sum_{\ell=1}^{r-1} x_{\ell}\left(F_{\ell 1}^{\ell}\left(\bar{x}_{r}\right)+p_{r 2 \ell 1}^{r \ell}\left(\bar{x}_{r}\right) x_{r}^{\tau}\right)+x_{r} F_{r 1}^{r}\left(\bar{x}_{r}\right) \in C
$$

for all $\bar{x}_{r} \in J_{2}^{r}$. By the induction hypothesis, there are a nonzero ideal $I$ of $R$ contained in $J_{2}$ and $(r-2)$-additive maps $p_{i 2 \ell 1}: I^{r-2} \rightarrow Q_{m l}(R)$ such that

$$
E_{i 2}^{i}\left(\bar{x}_{r}\right)=\sum_{\substack{\ell=1 \\ \ell \neq i}}^{r} x_{\ell} p_{i 2 \ell 1}^{i \ell}\left(\bar{x}_{r}\right)
$$

for all $\bar{x}_{r} \in I^{r}$ and $1 \leq i \leq r-1$. Thus (2.6) becomes

$$
\sum_{i=1}^{r} E_{i 1}^{i}\left(\bar{x}_{r}\right) x_{i}+\sum_{\ell=1}^{r} x_{\ell}\left(F_{\ell 1}^{\ell}\left(\bar{x}_{r}\right)+\sum_{\substack{i=1 \\ i \neq \ell}}^{r} p_{i 2 \ell 1}^{i \ell}\left(\bar{x}_{r}\right) x_{i}^{\tau}\right) \in C
$$


for all $\bar{x}_{r} \in I^{r}$. According to [1, Corollary 2.11], there exist $(r-2)$-additive maps $p_{i 1 \ell 1}: I^{r-2} \rightarrow Q_{m l}(R)$ and $(r-1)$-additive maps $\lambda_{i 1}: I^{r-1} \rightarrow C$ such that

$$
E_{i 1}^{i}\left(\bar{x}_{r}\right)=\sum_{\substack{1 \leq \ell \leq r \\ \ell \neq i}} x_{\ell} p_{i 1 \ell 1}^{i \ell}\left(\bar{x}_{r}\right)+\lambda_{i 1}^{i}\left(\bar{x}_{r}\right)
$$

and

$$
F_{\ell 1}^{\ell}\left(\bar{x}_{r}\right)+\sum_{\substack{i=1 \\ i \neq \ell}}^{r} p_{i 2 \ell 1}^{i \ell}\left(\bar{x}_{r}\right) x_{i}^{\tau}=-\sum_{\substack{1 \leq i \leq r \\ i \neq \ell}} p_{i 1 \ell 1}^{i \ell}\left(\bar{x}_{r}\right) x_{i}-\lambda_{\ell 1}^{\ell}\left(\bar{x}_{r}\right)
$$

for all $\bar{x}_{r} \in I^{r}$, where $1 \leq i, \ell \leq r$, as asserted.

Note that if $\delta: R \rightarrow Q_{m s}(R)$ is a Jordan $\tau$-derivation, then

$$
\delta(x y+y x)=\delta(x) y^{\tau}+y \delta(x)+\delta(y) x^{\tau}+x \delta(y)
$$

for all $x, y \in R$.

We are now ready to prove Theorem 1.2 .

Proof of Theorem 1.2. According to [16, Theorem 2.9], we can assume that $R$ is not a PI-ring. Let $\delta: R \rightarrow Q_{m s}(R)$ be a Jordan $\tau$-derivation. Define the bi-additive map $B: R \times R \rightarrow Q_{m s}(R)$ by $B(x, y)=\delta(x y+y x)$ for $x, y \in R$. It follows from [14, Lemma 2.3] that

$$
B(x w, y z)-B(x, w y z)=B(z x w, y)-B(z x, w y)
$$

for all $x, y, z, w \in R$. So, by (2.7), we have

$$
\begin{aligned}
& \left(\delta(y z) w^{\tau}-\delta(w y z)\right) x^{\tau}+\left(\delta(x w) z^{\tau}-\delta(z x w)\right) y^{\tau} \\
+ & \left(\delta(w y) x^{\tau}-\delta(y) w^{\tau} x^{\tau}\right) z^{\tau}+\left(\delta(z x) y^{\tau}-\delta(x) z^{\tau} y^{\tau}\right) w^{\tau} \\
+ & x(w \delta(y z)-\delta(w y z))+y(z \delta(x w)-\delta(z x w)) \\
+ & z(x \delta(w y)-x w \delta(y))+w(y \delta(z x)-y z \delta(x))=0
\end{aligned}
$$

for all $x, y, z, w \in R$. According to Corollary 2.2 , there exist a nonzero ideal $I_{1}$ of $R$ and bi-additive maps $r_{13}, r_{23}, r_{43}: I_{1}^{2} \rightarrow Q_{m l}(R)$ such that

$$
x(\delta(w y)-w \delta(y))=-r_{13}(y, w) x^{\tau}-r_{23}(x, w) y^{\tau}-r_{43}(x, y) w^{\tau}
$$

for all $x, y, w \in I_{1}$. Again, there are additive maps $p, q: I_{2} \rightarrow Q_{m l}(R)$ so that

$$
\delta(w y)-w \delta(y)=-p(y) w^{\tau}-q(w) y^{\tau}
$$

for all $y, w \in I_{2}$, where $I_{2}$ is a nonzero ideal of $R$ contained in $I_{1}$. Let $x, y, t \in I_{2}$. We have

$$
\delta(x y)=x \delta(y)-p(y) x^{\tau}-q(x) y^{\tau}
$$


Replacing $x$ by $t x$ in 2.8 , we obtain

$$
\delta(t x y)=t x \delta(y)-p(y) x^{\tau} t^{\tau}-q(t x) y^{\tau} .
$$

Left-multiplying (2.8) by $t$, we get $t \delta(x y)=t x \delta(y)-t p(y) x^{\tau}-t q(x) y^{\tau}$. Thus,

$$
\delta(t x y)-t \delta(x y)=(t q(x)-q(t x)) y^{\tau}-p(y) x^{\tau} t^{\tau}+t p(y) x^{\tau} .
$$

Replacing $x, y$ by $t, x y$ respectively in $(2.8)$, we have

$$
\delta(t x y)-t \delta(x y)=-p(x y) t^{\tau}-q(t) y^{\tau} x^{\tau} .
$$

Comparing the two equalities above, we see that

$$
(t q(x)-q(t x)) y^{\tau}+\left(p(x y)-p(y) x^{\tau}\right) t^{\tau}+\left(t p(y)+q(t) y^{\tau}\right) x^{\tau}=0
$$

for all $x, y, t \in I_{2}$. By Lemma 2.3 .

$$
t q(x)=q(t x), \quad p(x y)=p(y) x^{\tau} \quad \text { and } \quad t p(y)=-q(t) y^{\tau}
$$

for all $x, y, t \in I_{3}$, where $I_{3}$ is a nonzero ideal of $R$ contained in $I_{2}$. According to 11 , Lemma 2.1], there is $a \in Q_{m l}(R)$ such that $q(x)=x a$ for $x \in I_{3}$. So

$$
t p(y)=-q(t) y^{\tau}=-t a y^{\tau}
$$

for $t, y \in I_{3}$, i.e., $I_{3}\left(p(y)+a y^{\tau}\right)=0$ for all $y \in I_{3}$. Thus, $p(y)=-a y^{\tau}$ and it follows from (2.8) that

$$
\delta(x y)-x \delta(y)=a y^{\tau} x^{\tau}-x a y^{\tau}
$$

for $x, y \in I_{3}$. Let $\widetilde{\delta}: I_{3} \rightarrow Q_{m l}(R)$ be defined by $\widetilde{\delta}(x)=a x^{\tau}-x a$ for all $x \in I_{3}$. Then $\widetilde{\delta}(x y)=a y^{\tau} x^{\tau}-x y a$ and

$$
(\widetilde{\delta}-\delta)(x y)=x a y^{\tau}-x y a-x \delta(y)=x(\widetilde{\delta}-\delta)(y)
$$

for $x, y \in I_{3}$. So there exists $c \in Q_{m l}(R)$ such that $(\widetilde{\delta}-\delta)(x)=x c$ for all $x \in I_{3}$ (see [11, Lemma 2.1]). Define $J:=\widetilde{\delta}-\delta$, a Jordan $\tau$-derivation of $I_{3}$. Thus, $x^{2} c=$ $J\left(x^{2}\right)=x J(x)+J(x) x^{\tau}=x^{2} c+x c x^{\tau}$ for all $x \in I_{3}$; that is, $x c x^{\tau}=0$ for all $x \in I_{3}$. By [5, Lemma 2.2], $c=0$ follows, i.e., $\delta=\widetilde{\delta}$ on $I_{3}$. Therefore, $\delta(x)=a x^{\tau}-x a$ for $x \in I_{3}$. By [16, Lemma 2.6], $a \in Q_{m s}(R)$. Finally, we will show that $\delta$ is X-inner. Let $x \in I_{3}$ and $y \in R$. Then

$$
\delta(x y+y x)=a y^{\tau} x^{\tau}+a x^{\tau} y^{\tau}-x y a-y x a
$$

and

$$
\begin{aligned}
\delta(x y+y x) & =\delta(x) y^{\tau}+y \delta(x)+\delta(y) x^{\tau}+x \delta(y) \\
& =a x^{\tau} y^{\tau}-x a y^{\tau}+y a x^{\tau}-y x a+\delta(y) x^{\tau}+x \delta(y) .
\end{aligned}
$$


Comparing these equations, we have

$$
\left(\delta(y)-\left(a y^{\tau}-y a\right)\right) x^{\tau}+x\left(\delta(y)-\left(a y^{\tau}-y a\right)\right)=0
$$

for all $x \in I_{3}$ and $y \in R$. Fix $y \in R$ and set $q:=\delta(y)-\left(a y^{\tau}-y a\right)$. Then, for $x, z \in I_{3}$,

$$
x z q=-q(x z)^{\tau}=-q z^{\tau} x^{\tau}=z q x^{\tau}=-z x q
$$

and so $(x z+z x) q=0$ for all $x, z \in I_{3}$. This implies $(x z+z x) q=0$ for all $x, z \in Q_{m l}(R)$. If char $R \neq 2$, let $z=1$ and so $2 x q=0$ for all $x \in Q_{m l}(R)$ implying $q=0$. If char $R=2$, then $\left[Q_{m l}(R), Q_{m l}(R)\right] q=0$ forcing $q=0$. Hence $\delta(y)=a y^{\tau}-y a$ for all $y \in R$, as desired.

\section{Proof of Theorem 1.4}

Throughout the section, $R$ is a prime GPI-ring with an anti-automorphism $\tau$ of the first kind. Let $u \in Q_{s}(R)$ be fixed such that $x^{\tau^{2}}=u x u^{-1}$ for all $x \in R$.

Lemma 3.1. $u^{\tau} u=u u^{\tau} \in C$.

Proof. Let $x \in R$. Then

$$
u^{\tau} u x=u^{\tau} x^{\tau^{2}} u=\left(x^{\tau} u\right)^{\tau} u=\left(u\left(x^{\tau}\right)^{\tau^{-2}}\right)^{\tau} u=x u^{\tau} u .
$$

Hence $u^{\tau} u=u u^{\tau} \in C$.

Now, by Lemma 3.1, we fix $\beta:=u^{\tau} u=u u^{\tau} \in C$ and so $u^{\tau}=\beta u^{-1}$. Since $R$ is a prime GPI-ring, $R C$ is a primitive ring with nonzero socle and so $Q_{m s}(R C)=Q_{s}(R C)$. In view of [5. Theorem 1.2], the aim of this section is to extend $\delta$ to a Jordan $\tau$-derivation of $R C$ when char $R \neq 2$. The following result plays a key role.

Lemma 3.2. Let $f: R \rightarrow Q_{m l}(R)$ be an additive map such that

$$
x f(y)+f(y) x^{\tau}=y f(x)+f(x) y^{\tau}
$$

for all $x, y \in R$. If $\operatorname{deg} \tau^{2}>2$, then $f=0$ on some nonzero ideal $I$ of $R$.

Proof. Choose a nonzero ideal such that $u I_{1} \subseteq R$. Then, by replacing $x$ with $u x$ in (3.1),

$$
u x f(y) u+\beta f(y) x^{\tau}=y f(u x) u+f(u x) y^{\tau} u
$$

for $x \in I_{1}$. Also, by 3.1 ,

$$
u x f(y) u+u f(y) x^{\tau} u=u y f(x) u+u f(x) y^{\tau} u
$$


Comparing the two equations, we have

$$
\beta f(y) x^{\tau}-u f(y) x^{\tau} u=y f(u x) u-u y f(x) u+(f(u x)-u f(x)) y^{\tau} u
$$

for $x \in I_{1}$. Replacing $y$ by $u y$ in 3.2 ,

$$
\beta f(u y) x^{\tau}-u f(u y) x^{\tau} u=u y f(u x) u-u^{2} y f(x) u+\beta(f(u x)-u f(x)) y^{\tau} .
$$

Also, by (3.2),

$$
u \beta f(y) x^{\tau}-u^{2} f(y) x^{\tau} u=u y f(u x) u-u^{2} y f(x) u+u(f(u x)-u f(x)) y^{\tau} u .
$$

Comparing the two equations, we have

$$
\begin{aligned}
& \beta(f(u y)-u f(y)) x^{\tau}-u(f(u y)-u f(y)) x^{\tau} u \\
= & \beta(f(u x)-u f(x)) y^{\tau}-u(f(u x)-u f(x)) y^{\tau} u
\end{aligned}
$$

for all $x, y \in I_{1}$. By [1, Theorem 2.5], $f(u y)=u f(y)$ for all $y \in I_{1}$. So $(3.2)$ becomes

$$
\beta f(y) x^{\tau}-u f(y) x^{\tau} u=y u f(x) u-u y f(x) u
$$

for all $x, y \in I_{1}$. Choose a nonzero ideal $I_{2}$ of $R$ contained in $I_{1}$ such that $u I_{2} \subseteq I_{1}$. Replacing $x$ by $u x$ in 3.3 , we have

$$
\beta^{2} f(y) x^{\tau}-\beta u f(y) x^{\tau} u=y u^{2} f(x) u^{2}-u y u f(x) u^{2}
$$

for all $x, y \in I_{2}$. On the other hand, 3.3 implies

$$
\beta^{2} f(y) x^{\tau}-\beta u f(y) x^{\tau} u=\beta(y u f(x) u-u y f(x) u)
$$

for all $x, y \in I_{2}$. Comparing the two equations, we have

$$
y\left(\beta u f(x)-u^{2} f(x) u\right)+u y(u f(x) u-\beta f(x))=0
$$

for all $x, y \in I_{2}$. Similarly, $u f(x) u=\beta f(x)$ for all $x \in I_{2}$. Thus (3.3) becomes

$$
\beta f(y) x^{\tau}-u f(y) x^{\tau} u=\beta y f(x)-u y f(x) u
$$

for all $x, y \in I_{2}$. Choose a nonzero ideal $I_{3}$ of $R$ contained in $I_{2}$ such that $I_{3} u \subseteq I_{2}$. Replacing $y$ by $y u$ in (3.4,

$$
\begin{aligned}
\beta f(y u) x^{\tau}-u f(y u) x^{\tau} u & =\beta y u f(x)-u y u f(x) u=\beta^{2} y f(x) u^{-1}-\beta u y f(x) \\
& =\beta(\beta y f(x)-u y f(x) u) u^{-1}=\beta^{2} f(y) x^{\tau} u^{-1}-\beta u f(y) x^{\tau}
\end{aligned}
$$

for all $x, y \in I_{3}$. So we get

$$
\beta^{2} f(y) x^{\tau}-(\beta u f(y)+\beta f(y u)) x^{\tau} u+u f(y u) x^{\tau} u^{2}=0
$$

for all $x, y \in I_{3}$. By [1, Theorem 2.5], $f(y)=0$ for all $y \in I_{3}$, as desired. 
Remark 3.3. In Lemma 3.2, the case for $\operatorname{deg} \tau^{2}=1$ had been solved by Beidar and Martindale III (see [1]). However, the solution of (3.1) is still unknown when $\operatorname{deg} \tau^{2}=2$. If we can solve it, then the same result holds for $\operatorname{deg} \tau^{2}=2$ in Theorem 1.4

Lemma 3.4. Suppose $\operatorname{deg} \tau^{2}>2$. Then, for each $\alpha \in C$, there exists an nonzero ideal $I$ of $R$ such that $\alpha I \subseteq R$ and $\delta(\alpha x)=\alpha \delta(x)$ for all $x \in I$.

Proof. Choose a nonzero ideal $I_{1}$ of $R$ such that $\alpha I_{1} \subseteq R$. Let $x, y \in I_{1}$. Then

$$
\delta((\alpha x) y+y(\alpha x))=\delta(\alpha x) y^{\tau}+y \delta(\alpha x)+\alpha \delta(y) x^{\tau}+\alpha x \delta(y)
$$

and

$$
\delta(x(\alpha y)+(\alpha y) x)=\alpha \delta(x) y^{\tau}+\alpha y \delta(x)+\delta(\alpha y) x^{\tau}+x \delta(\alpha y) .
$$

Comparing the two equations, we have

$$
(\delta(\alpha y)-\alpha \delta(y)) x^{\tau}+x(\delta(\alpha y)-\alpha \delta(y))=(\delta(\alpha x)-\alpha \delta(x)) y^{\tau}+y(\delta(\alpha x)-\alpha \delta(x)) .
$$

By Lemma 3.2, there is a nonzero ideal $I$ of $R$ contained in $I_{1}$ such that $\delta(\alpha x)=\alpha \delta(x)$ for all $x \in I$.

Lemma 3.5. Suppose $\operatorname{deg} \tau^{2}>2$. Then every Jordan $\tau$-derivation $\delta$ of $R$ can be extended to a Jordan $\tau$-derivation of $R C$.

Applying Lemma 3.5, we are now ready to prove Theorem 1.4 .

Proof of Theorem 1.4. In view of [16. Theorem 2.9], we can assume that $R$ is not a PIring. By [13, Theorem 1.2], we also assume that $\operatorname{deg} \tau^{2}>2$. Recall that if $R$ is a prime GPI-ring and $R C$ is a division ring, then $R$ is a PI-ring. So $R C$ is not a division ring. Let $\delta: R \rightarrow Q_{m s}(R)$ be a Jordan $\tau$-derivation of $R$. Since the socle of $R C$ is nonzero, $Q_{m s}(R C)=Q_{s}(R C)$. By Lemma 3.5, $\delta$ can be extended to a Jordan $\tau$-derivation $\widetilde{\delta}: R C \rightarrow Q_{s}(R C)$ of $R C$. According to 5 , Theorem 1.2], there exists $a \in Q_{s}(R C)$ such that $\widetilde{\delta}(x)=a x^{\tau}-x a$ for $x \in R C$. In particular, $\delta(x)=a x^{\tau}-x a$ for all $x \in R$. As a consequence of [2, Proposition 2.1.10], $Q_{m s}(R)=Q_{m s}(R C)=Q_{s}(R C)$ and so $a \in Q_{m s}(R)$. Hence $\delta$ is $\mathrm{X}$-inner, as desired.

Proof of Lemma 3.5. Choose a subset $\left\{w_{i}\right\}_{i \in \Phi}$ of $R$ which is a basis of $R C$ over $C$, where $\Phi$ is a nonempty well-ordered set. Then any element of $R C$ can be written as the form $\sum_{i \in \Phi} \alpha_{i} w_{i}$, where $\alpha_{i}=0$ for all but finitely many $i \in \Phi$. Recall that $Q_{m s}(R)=Q_{m s}(R C)$. Define $\widetilde{\delta}: R C \rightarrow Q_{m s}(R C)$ by

$$
\widetilde{\delta}\left(\sum_{i \in \Phi} \alpha_{i} w_{i}\right)=\sum_{i \in \Phi} \alpha_{i} \delta\left(w_{i}\right) .
$$


Then it is clearly a well-defined additive map since $\left\{w_{i}\right\}_{i \in \Phi}$ forms a basis of $R C$ over $C$. We claim that $\left.\widetilde{\delta}\right|_{R}=\delta$. Let $x \in R$. Write $x=\sum_{i \in \Phi} \alpha_{i} w_{i}$. By Lemma 3.4, there is a nonzero ideal $I$ of $R$ such that $\alpha_{i} I \subseteq R$ and $\delta\left(\alpha_{i} y\right)=\alpha_{i} \delta(y)$ for all $i \in \Phi$ and $y \in I$. Let $y \in I$. Then

$$
\begin{aligned}
\delta(x y+y x) & =\sum_{i \in \Phi} \delta\left(\left(\alpha_{i} y\right) w_{i}+w_{i}\left(\alpha_{i} y\right)\right) \\
& =\sum_{i \in \Phi} \delta\left(\alpha_{i} y\right) w_{i}^{\tau}+\delta\left(w_{i}\right) \alpha_{i} y^{\tau}+w_{i} \delta\left(\alpha_{i} y\right)+\alpha_{i} y \delta\left(w_{i}\right) \\
& =\delta(y) x^{\tau}+x \delta(y)+\sum_{i \in \Phi}\left(\alpha_{i} \delta\left(w_{i}\right) y^{\tau}+\alpha_{i} y \delta\left(w_{i}\right)\right)
\end{aligned}
$$

Comparing this with (2.7), we have

$$
\left(\delta(x)-\sum_{i \in \Phi} \alpha_{i} \delta\left(w_{i}\right)\right) y^{\tau}+y\left(\delta(x)-\sum_{i \in \Phi} \alpha_{i} \delta\left(w_{i}\right)\right)=0
$$

for all $y \in I$. By applying the same argument as the last part of the proof of Theorem 1.2 , we have $\delta(x)=\sum_{i \in \Phi} \alpha_{i} \delta\left(w_{i}\right)=\widetilde{\delta}(x)$ and so the claim holds.

Finally we show that $\widetilde{\delta}$ is a Jordan $\tau$-derivation. Let $x=\sum_{i \in \Phi} \alpha_{i} w_{i} \in R C$. For each $i, j \in \Phi$, write $w_{i} w_{j}=\sum_{k \in \Phi} \gamma_{k}^{i j} w_{k} \in R$, where $\gamma_{k}^{i j} \in C$. Then

$$
\begin{aligned}
\widetilde{\delta}\left(x^{2}\right) & =\widetilde{\delta}\left(\sum_{i, j} \alpha_{i} \alpha_{j} w_{i} w_{j}\right)=\widetilde{\delta}\left(\sum_{i, j} \alpha_{i} \alpha_{j} \sum_{k \in \Phi} \gamma_{k}^{i j} w_{k}\right)=\sum_{i, j} \alpha_{i} \alpha_{j} \sum_{k \in \Phi} \gamma_{k}^{i j} \delta\left(w_{k}\right) \\
& =\sum_{i, j} \alpha_{i} \alpha_{j} \widetilde{\delta}\left(w_{i} w_{j}\right)=\sum_{i, j} \alpha_{i} \alpha_{j} \delta\left(w_{i} w_{j}\right)=\sum_{i} \alpha_{i}^{2} \delta\left(w_{i}^{2}\right)+\sum_{i<j} \alpha_{i} \alpha_{j} \delta\left(w_{i} w_{j}+w_{j} w_{i}\right) \\
& =\sum_{i} \alpha_{i}^{2}\left(w_{i} \delta\left(w_{i}\right)+\delta\left(w_{i}\right) w_{i}^{\tau}\right)+\sum_{i<j} \alpha_{i} \alpha_{j}\left(\delta\left(w_{i}\right) w_{j}^{\tau}+w_{j} \delta\left(w_{i}\right)+\delta\left(w_{j}\right) w_{i}^{\tau}+w_{i} \delta\left(w_{j}\right)\right) \\
& =x \widetilde{\delta}(x)+\widetilde{\delta}(x) x^{\tau} .
\end{aligned}
$$

Hence the proof of Lemma 3.5 is complete.

\section{Acknowledgments}

The author thanks Professor Tsiu-Kwen Lee for useful suggestions, and thanks the referee for carefully reading the manuscript and for giving helpful comments. The work was supported in part by the Ministry of Science and Technology of Taiwan (MOST 107-2115M-002-018-MY2 due to Prof. T.-K. Lee) and the National Center for Theoretical Sciences, Taipei Office. 


\section{References}

[1] K. I. Beidar and W. S. Martindale III, On functional identities in prime rings with involution, J. Algebra 203 (1998), no. 2, 491-532.

[2] K. I. Beidar, W. S. Martindale III and A. V. Mikhalev, Rings with Generalized Identities, Monographs and Textbooks in Pure and Applied Mathematics 196, Marcel Dekker, New York, 1996.

[3] M. Brešar, Jordan derivations on semiprime rings, Proc. Amer. Math. Soc. 104 (1988), no. 4, 1003-1006.

[4] M. Brešar and J. Vukman, On some additive mappings in rings with involution, Aequationes Math. 38 (1989), no. 2-3, 178-185.

[5] C.-L. Chuang, A. Fošner and T.-K. Lee, Jordan $\tau$-derivations of locally matrix rings, Algebr. Represent. Theory 16 (2013), no. 3, 755-763.

[6] J. M. Cusack, Jordan derivations on rings, Proc. Amer. Math. Soc. 53 (1975), no. 2, $321-324$.

[7] A. Fošner and T.-K. Lee, Jordan *-derivations of finite-dimensional semiprime algebras, Canad. Math. Bull. 57 (2014), no. 1, 51-60.

[8] V. K. Harčenko, Generalized identities with automorphisms, Algebra i Logika 14 (1975), no. 2, 215-237.

[9] I. N. Herstein, Jordan derivations of prime rings, Proc. Amer. Math. Soc. 8 (1957), 1104-1110.

[10] W. S. Martindale III, Prime rings satisfying a generalized polynomial identity, J. Algebra 12 (1969), 576-584.

[11] T.-K. Lee, Generalized skew derivations characterized by acting on zero products, Pacific J. Math. 216 (2004), no. 2, 293-301.

[12] _ Jordan $\sigma$-derivations of prime rings, Rocky Mountain J. Math. 47 (2017), no. $2,511-525$.

[13] T.-K. Lee, T.-L. Wong and Y. Zhou, The structure of Jordan *-derivations of prime rings, Linear Multilinear Algebra 63 (2015), no. 2, 411-422.

[14] T.-K. Lee and Y. Zhou, Jordan *-derivations of prime rings, J. Algebra Appl. 13 (2014), no. 4, 1350126, 9 pp. 
[15] T.-K. Lee and J.-H. Lin, Jordan derivations of prime rings with characteristic two, Linear Algebra Appl. 462 (2014), 1-15.

[16] _ Jordan $\tau$-derivations of prime rings, Comm. Algebra 43 (2015), no. 12, $5195-5204$.

[17] P. Šemrl, On Jordan *-derivations and an application, Colloq. Math. 59 (1990), no. 2, $241-251$.

[18] _ Quadratic functionals and Jordan *-derivations, Studia Math. 97 (1991), no. $3,157-165$.

[19] Q Quadratic and quasi-quadratic functionals, Proc. Amer. Math. Soc. 119 (1993), no. 4, 1105-1113.

[20] J Jordan *-derivations of standard operator algebras, Proc. Amer. Math. Soc. 120 (1994), no. 2, 515-518.

Jheng-Huei Lin

Department of Mathematics, National Taiwan University, Taipei, Taiwan

E-mail address: r01221012@ntu.edu.tw 